\title{
High-contrast optical imaging of companions: the case of the brown dwarf binary HD 130948 BC
}

\author{
L. Labadie ${ }^{1,2}$, R. Rebolo ${ }^{1,6}$, I. Villó ${ }^{3}$, J. A. Pérez-Prieto ${ }^{1}$, A. Pérez-Garrido ${ }^{3}$, S. R. Hildebrandt ${ }^{4}$, B. Femenía ${ }^{1,2}$, \\ A. Díaz-Sanchez ${ }^{3}$, V. J. S. Béjar ${ }^{1,2}$, A. Oscoz ${ }^{1}$, R. López $^{1}$, J. Piqueras ${ }^{5}$, and L. F. Rodríguez ${ }^{1}$ \\ ${ }^{1}$ Instituto de Astrofisica de Canarias, C/ Via Lactea s/n, La Laguna, 38200 Tenerife, Spain \\ e-mail: labadie@iac.es \\ 2 Departamento de Astrofisica, Universidad de La Laguna, 38205 La Laguna, Tenerife, Islas Canarias, Spain \\ 3 Universidad Politecnica de Cartagena, Campus Muralla del Mar, Cartagena, 30202 Murcia, Spain \\ ${ }^{4}$ Laboratoire de Physique Subatomique et de Cosmologie, 53 avenue des Martyrs, 38026 Grenoble, France \\ 5 Max-Planck-Institut für Sonnensystemforschung, Max-Planck-Str. 2, 37191 Katlenburg-Lindau, Germany \\ ${ }^{6}$ Consejo Superior de Investigaciones Cientificas, Spain
}

Received 5 March 2010 / Accepted 27 September 2010

\begin{abstract}
Context. High-contrast imaging at optical wavelengths is limited by the modest correction of conventional near-IR optimized AO systems. We take advantage of new fast and low-readout-noise detectors to explore the potential of fast imaging coupled to postprocessing techniques to detect faint companions of stars at small angular separations.

Aims. We have focused on I-band direct imaging of the previously detected brown dwarf binary HD 130948 BC, attempting to spatially resolve the $\mathrm{L} 2+\mathrm{L} 2$ system considered as a benchmark for the determination of substellar objects dynamical masses.

Methods. We used the lucky-imaging instrument FastCam at the 2.5-m Nordic Telescope to obtain quasi diffraction-limited images of HD 130948 with $\sim 0.1^{\prime \prime}$ resolution. In order to improve the detectability of the faint binary in the vicinity of a bright $(I=5.19 \pm 0.03)$ solar-type star, we implemented a post-processing technique based on wavelet transform filtering of the image, which allows us to strongly enhance the presence of point-like sources in regions where the primary halo generally dominates.

Results. We detect for the first time the binary brown dwarf HD 130948 BC in the optical band $I$ with a SNR $\sim 9$ at $2.561^{\prime \prime} \pm 0.007^{\prime \prime}$ (46.5 AU) from HD 130948 A and confirm in two independent datasets (2008 May 29 and July 25) that the object is real, as opposed to time-varying residual speckles. We do not resolve the binary, which can be explained by astrometric results posterior to our observations, which predict a separation below the telescope resolution. We reach a contrast of $\Delta I=11.30 \pm 0.11$ at this distance, and estimate a combined magnitude for this binary $I=16.49 \pm 0.11$ and a $I-J$ color of $3.29 \pm 0.13$. At $1^{\prime \prime}$, we reach a detectability 10.5 mag fainter than the primary after image post-processing.

Conclusions. We obtain on-sky validation of a technique based on speckle imaging and wavelet-transform post-processing, which improves the high-contrast capabilities of speckle imaging. The $I-J$ color measured for the BD companion is slightly bluer, but still consistent with what is typically found for L2 dwarfs ( 3.4-3.6).
\end{abstract}

Key words. instrumentation: high angular resolution - methods: observational - techniques: image processing - binaries: close brown dwarfs - circumstellar matter

\section{Introduction}

A direct determination of dynamical masses of very low-mass (VLM) objects is essential to calibrate the mass-luminosity relationship. This is particularly relevant for understanding the brown dwarfs (BDs) evolution. Dynamical masses can be determined by observing close multiple BD systems (Zapatero Osorio et al. 2004; Bouy et al. 2004; Stassun et al. 2006; Dupuy et al. 2009). Brown dwarfs close binaries with orbital periods $\lesssim 10 \mathrm{yr}$ represent a valuable sample for a model-independent mass determination within a realistic time baseline. Observationally, this requires to spatially resolve the binary, which also permits us to obtain a direct measurement of the flux of each component. Because BD systems are also detected as close companions to bright main-sequence stars, another difficulty resides in the strong contrast needed to detect them (cf. the case HR $7672 \mathrm{~B}$ in Liu et al. 2002), on top of the detectability issue because of their intrinsic low luminosity. So far, the sample of such companion BD binaries is limited to a few (Burgasser et al. 2005), mostly characterized in the near-IR with the help of 8$10 \mathrm{~m}$ class telescopes.

Optical data are necessary for a full characterization of the spectral energy distribution, which is key to the determination of effective temperatures and bolometric luminosity. In the visible domain, close binaries can be spatially resolved with speckle imaging (Law et al. 2006a), a technique that delivers diffractionlimited optical counterpart to AO-assisted infrared images. The question of high contrast in speckle imaging has been investigated in the past by Boccaletti et al. (2001) using the "dark speckles" method as an additional stage of cleaning to improve the detectability of faint companions. Coupled to the adaptive optics system ADONIS and a Lyot stellar coronograph, these authors obtained K-band contrasts of $1.5-4.5 \times 10^{-3}\left(\Delta m_{K} \sim 6-7\right)$ at $0.5-0.9^{\prime \prime}$.

In this paper, we have focused on the brown dwarf binary HD 130948 BC, originally reported by Potter et al. (2002). As 
part of a restricted sample of BD binaries companion to a solartype star, HD 130948 BC is a unique benchmark for the study of the mass, luminosity, and age of L-type substellar objects. Lying at $\sim 45$ AU from HD $130948 \mathrm{~A}$, the determination of the close BD binary orbit based on infrared AO images permitted Dupuy et al. (2009) to derive a total mass estimated as $0.109 \pm 0.03 M_{\odot}$. We intent to use speckle imaging techniques to conduct a first optical ground-based high-resolution study of this object in order to extend its physical characterization to shorter wavelengths, and at the same time using it as an observational testbench for diffraction-limited imaging from the ground at visible wavelengths. Here, we report first $I$-band high-contrast speckle imaging of the HD 130948 system. The detection of the BD companion is improved through image post-processing. This emphasizes how small to medium size telescopes can still be exploited for high-angular and high-contrast imaging. In Sect. 2 we present our observations and the data reduction procedure. In Sect. 3 we present our imaging, astrometric, and photometric results for HD 130948 BC, briefly discussing our derived $I-J$ color.

\section{Observations and data reduction}

\subsection{Imaging}

HD 130948 was observed on the nights of 2008 May 29 and July 25 with the FastCam instrument (Oscoz et al. 2008) installed at the 2.5-m Nordic Telescope (NOT) at the Roque de los Muchachos Observatory, Spain. Observing conditions during these nights were good with an average seeing of $0.5^{\prime \prime}$ in the $I$-band and clear weather. In brief, FastCam is an optical imager based on a conventional low-noise CCD camera from Andor Technology, which allows us to record speckle-featuring unsaturated images at a rate of several tens of frames per second. Each frame captures a different pattern introduced by the atmospheric turbulence, where each speckle represents a diffraction-limited image of the source of interest.

FastCam was installed on the Cassegrain focus of the NOT. The pixel scale, determined from astrometric measurements in the M 15 globular cluster, is estimated at $31.17 \pm 0.03$ mas/pixel. The field-of-view accessible with the $512 \times 512 \mathrm{CCD}$ array is $16^{\prime \prime} \times 16^{\prime \prime}$. The raw data acquired by FastCam are composed of cubes of 1000 images each, with an individual integration time of $10-50 \mathrm{~ms}$. A total of 100 cubes (i.e. $10^{5}$ frames) and 50 cubes was acquired for HD 130948 in the nights of July 25 and May 29, respectively, as well as flat and dark images for standard image correction. After finding the brightest speckle in each image of the cube, we can select an arbitrary percent of images based on the brightest speckles sorted within the serie. Hence, those frames with the highest concentration of energy in a given speckle are selected. A subsequent shift-and-add process of the previously selected frames produces an image where the bright diffraction-limited core is surrounded by a fainter seeing halo. This technique is also referred to as lucky imaging (Law et al. 2006b). The resulting angular resolution then depends on the percentage of selected frames and, among others, on the natural seeing during the night and the integration time for each individual frame. The percent of selected frames results from a trade-off between a sufficiently high integration time and a good angular resolution. In our case, the individual integration time and the percent of selected frames were fixed to $30 \mathrm{~ms}$ and $30 \%$ respectively, leading to an effective total integration time of 900 s. Thus, FastCam delivered $\sim 0.1^{\prime \prime}$ resolution images, close to the diffraction-limit $1.22 \lambda / D$ of 84 mas of the NOT at $0.8 \mu \mathrm{m}$.
As in any imaging system aiming at compensating the effect of the atmospheric turbulence, the presence of the seeing halo in the images is a strong limitation to high-contrast capabilities. Since we are unable with the current observational technique to artificially reduce the starlight contribution in a way this can be done with a coronagraph, the flux contrast at a given distance from the star is improved by applying an additional post-processing stage to the image, which helps to suppress most of the seeing halo, unveiling fainter sources in the immediate vicinity of the star. We initially tried the classical solution of PSF-reference subtraction. But the strong time-variability of lucky-imaging PSF profiles at optical wavelengths prevents us from applying an efficient subtraction that would improve the detectability. Thus, we implemented a different approach based on the post-processing of the shift-and-add images in order to enhance particular spatial frequencies in the image. For this purpose, we have tested different image filtering algorithms, which are:

$\star$ the subtraction of a median box filtering.

$\star$ the implementation of a standard unsharp mask (i.e. $I=$ $I-I * g$, where $I$ is the input image, "*” denotes convolution and $g$ is a Gaussian kernel.

$\star$ the implementation of the wavelet transform of the shift-andadd image. All these algorithms, which are comparable to edge enhancement techniques, allow us to suppress a continuous offset (e.g. sky background, detector bias...) or an extended and diffused structure (e.g. a PSF halo) at a different spatial scale than the object of interest, hence favoring the detection of fainter point-like sources at higher spatial frequencies.

The best results were obtained with the unsharp mask filtering and the wavelet decomposition. In their numerical implementation, those two algorithms are very similar, but the wavelet algorithm gives the advantage of a multi-resolution approach. This last solution was finally selected and applied to our two different datasets of HD 130948, and is described in more detail in Appendix A.

\subsection{Photometry}

We used an absolute measurement of the HD 130948 A photometry to derive the $I$ magnitude of the companion from our images (see Sect. 3.4). In order to determine the $I$ magnitude of HD 130948 A, we carried out a dedicated photometric measurement, obtaining I-band images of HD 130948 on 2010 April 6 using the CAMELOT instrument mounted on the IAC80 telescope. This optical camera consists of a $2 \mathrm{k} \times 2 \mathrm{k}$ CCD detector with a $0.304^{\prime \prime} /$ pix plate scale, providing a field of view of $10.4 \times$ 10.4 arcmin. The same $I$ filter can be physically interchanged in FastCam and CAMELOT and match the Johnson-Cousins system. The spectral responses of each of the two detectors were obtained from available technical data, and the differential effect on a solar-type star spectrum was estimated to a residual magnitude of 2.9 milli-magnitude. We observed a serie of 10 images with an individual exposure time of $5 \mathrm{~s}$. Raw data were reduced by routines within the IRAF environment. Bias-images were subtracted using the overscan region and zero exposure time images, and flat-field correction was applied with dome flats. We performed aperture photometry with routines from the DAOPHOT package. We adopted an aperture of 5 FWHM (depending on the seeing conditions, this number varies between $6.4^{\prime \prime}$ to $8.7^{\prime \prime}$ ) for our photometric standard stars from Landolt (1992). This is a standard aperture recommended by several photometric manuals to avoid contamination. Because the HD 130948 images were defocused to avoid saturation, we adopted a larger aperture (10 FWHM) 

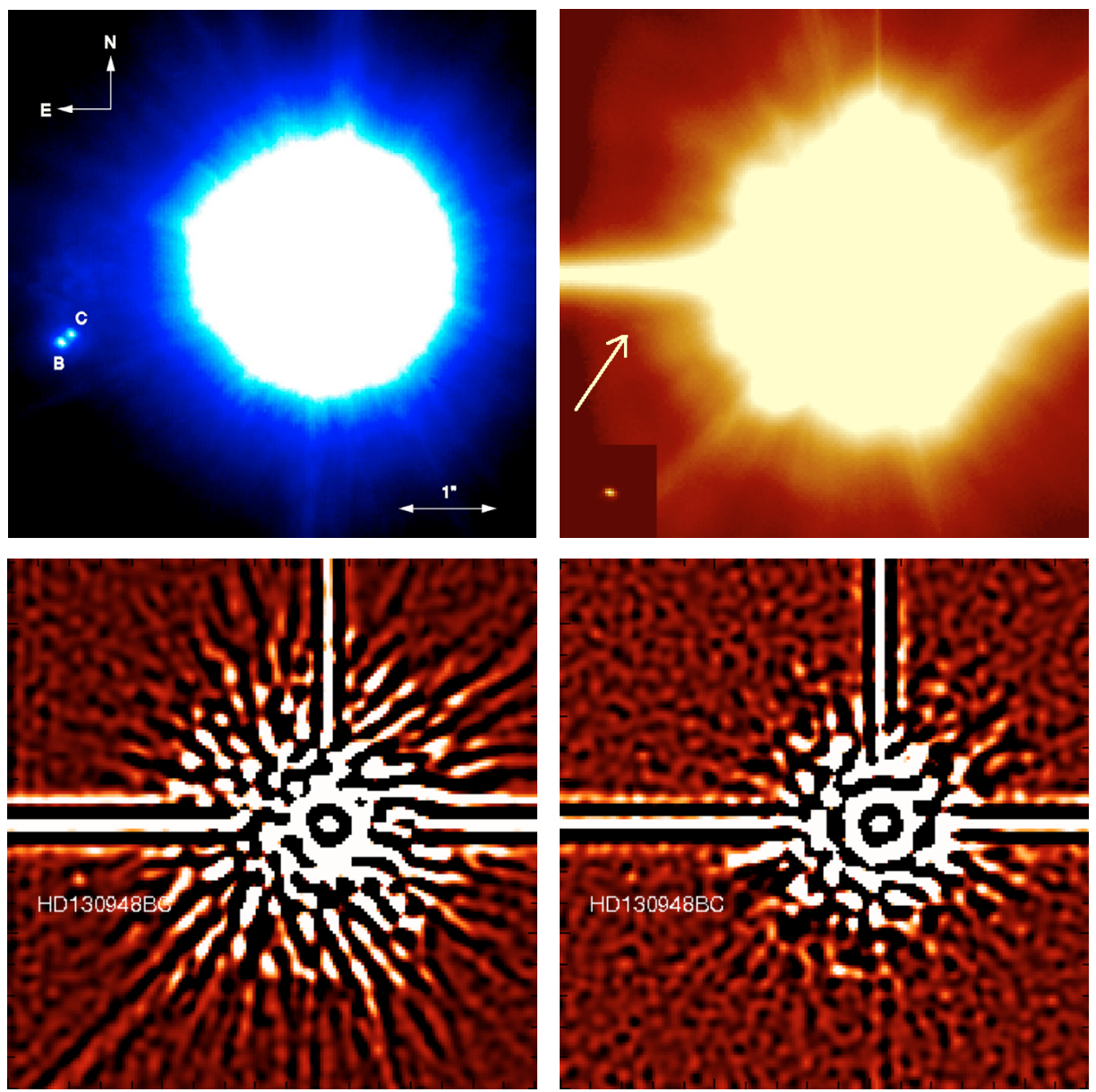

Fig. 1. Upper-left: original H-band detection of HD 130948 BC in Potter et al. (2002). Upper-right: direct lucky-imaging image resulting from the best $30 \%$ frames over a $10^{5}$ serie. The white arrow indicates the anticipated position of the companion. The inset shows the core of the PSF on the full intensity scale. Bottom-left: post-processed image from July 2008 revealing the BD binary companion, unresolved with the NOT at $0.8 \mu \mathrm{m}$. Bottom-right: post-processed image from May 2008 obtained with $5 \times 10^{4}$ frames. All four images have the same size. North is up, East is left. The effective total integration time to obtain the July image is $900 \mathrm{~s}$.

and therefore, in order to correct for the difference, we estimated the aperture correction from 5 to $10 F W H M$ with bright and isolated Landolt standard stars. We obtained an aperture correction of $0.033 \pm 0.005 \mathrm{mag}$, which was included in the photometry of our target. Weather conditions during our observations were photometric as assessed by observing photometric standard through the whole night, while the average seeing ranged from 1.3 to $2^{\prime \prime}$. In order to transform our instrumental magnitudes into apparent magnitudes, we observed four different Landolt standard star fields (each of them containing 3-6 standard stars) and repeated them during the night. We obtained 14 different images at 7 different pointings covering a range of airmasses from 1.1 to 2.1. We perform a linear fit to our data to obtain the zero points and the extinction coefficient, following the equation $i-I=a_{0}+k \times$ airmass, where $i$ and $I$ are, respectively, the instrumental magnitude and the apparent magnitude of the Landolt stars, $a_{0}$ is the zero point, $k$ is the extinction coefficient. We obtained $a_{0}=2.68 \pm 0.019(z \mathrm{mag}=25)$ and $k=0.17 \pm 0.013$. The error bars in the calibration were obtained from the estimated errors of the coefficients in the linear fit. Eventually, our correction from instrumental to apparent magnitudes for HD 130948 was finally $i-I=2.860 \pm 0.032(-0.033 \pm 0.005$ aperture correction $)=2.827 \pm 0.032$. The final error bar includes both the error in the calibration and in the instrumental magnitude.

\section{Results}

\subsection{Detection of the BD companion}

The panels of Fig. 1 show the imaging results from our observations. In the upper-left corner we display the original detection by Potter et al. (2002), in which the brown dwarf binary is resolved in the $H$-band with the $8-\mathrm{m}$ Gemini-North telescope. The upper-right corner shows the direct shift-and-add image of HD 130948 obtained with the data of July 25. In all the images, North is up, East is left. The FastCam images have been rotated by $90^{\circ}$ with respect to the original position of the detector on sky. The average full-width-at-half-maximum $(F W H M)$ is 131 mas, with a slight elongation toward the east (148 mas) against the north (114 mas). We attribute this effect to atmospheric dispersion because no ADC (atmospheric dispersion compensator) is available in the current FastCam+NOT configuration. The white arrow indicates the expected position of the $\mathrm{BD}$ companion with respect to HD $130948 \mathrm{~A}$, while the inset shows the core of the PSF on a different intensity scale.

The bottom part of Fig. 1 shows the HD 130948 system observed in July (left) and May 2008 (right) after the image filtering step. The brown dwarf is detected with a signal-to-noise ratio $(S N R) \sim 9$ and an average $F W H M \sim 110$ mas, at a position very consistent with earlier image (see next section). The 
BD binary HD 130948 B - HD 130948 C is unresolved in our image. According to the orbital solution derived by Dupuy et al. (2009), the BD separation at epoch 2008.56 is estimated to be 30 mas, which is below the theoretical diffraction-limit resolution of the NOT. The physical separation at the same epoch is $0.63 \mathrm{AU}$ assuming $d=18.17 \mathrm{pc}$ given by the same authors. Because the filtering process leaves speckles in the image that may mimic the presence of a companion, the detection is doubled-checked with a procedure that splits the 100 cubes of the July dataset into three separate datasets, which undergo the same post-processing step. In this way, speckle features simply resulting from the image processing are unlike to be repeated in the different sub-images, while a real companion remains detectable, although with a lower $S N R$. In our particular case, we also benefit from the additional dataset of May 29 (50 cubes), in which HD 130948 BC is searched for. In both cases, the BD companion binary is detected at the same location with respect to the G2V star. In the following part of this paper, the shift-andadd image of the best $30 \%$ selected frames and the filtered image will be referred as Image 1 and Image 2 respectively.

\subsection{Detectability and contrast curve}

In Fig. 2 we present the the 3- $\sigma$ detectability curve as a function of the distance to the central star for Image 1 and Image 2. These curves were obtained by estimating in the corresponding images the radial standard deviation profile in a $9 \times 9$ pixels slipping box averaged in azimuth over 22 angular positions in clean areas of the image (i.e. not affected by the vertical and horizontal spikes visible in Fig. 1) and separated by $5^{\circ}$. The gain is on the order of 2 mag when implementing the post-processing step. This result, interpreted in the perspective of the images of Fig. 1, suggests that the presence of the bright PSF halo in the direct image significantly contributes to the background noise at various spatial frequencies, degrading the detectability of a faint companion. On the contrary, in the filtered image the background noise associated to the bright halo is mostly filtered out around the spatial frequency constrained by the wavelet kernel. In other words, the wavelet filtering step strongly improves the signal-to-noise ratio of the object detection in absence of any external hardware system aiming at reducing the PSF halo (e.g. with a coronograph). In Fig. 2 we added a point showing the detection of HD 130948 BC (see next section for contrast estimation), which helps to better perceive the gain set by the postprocessing step.

\subsection{Astrometry}

To determine the separation between the primary and the BD binary, we first used the photo-center of Image 1 (or barycenter in a $15 \times 15$ pixels aperture centered on the primary) as the astrometric reference because we benefitted from an excellent signal-to-noise in the unsaturated image of the primary. The position of the photo-center coincides with the brightest pixel within 0.09 pixels. The same result was obtained using a twodimensional Gaussian fit of the primary PSF, which is explained by the high $S N R$ obtained on this bright object. On the contrary, we only implemented the Gaussian fit technique to determine the position of the faint companion in the filtered image, which proved to be more accurate than the photo-center technique for lower $S N R$. We first checked by simulating several artificial companions at similar distance and with comparable brightness to $\mathrm{HD} 130948 \mathrm{BC}$ that the presence of such a

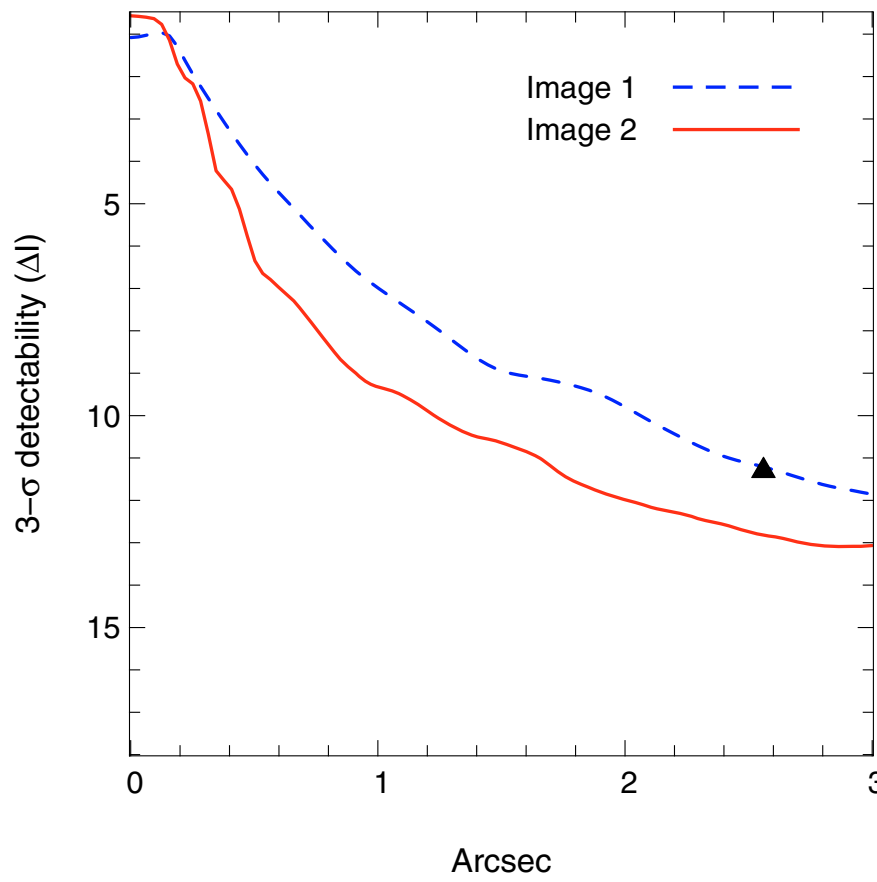

Fig. 2. 3- $\sigma$ detectability curve estimated in the two cases of Image 1 (dashed line) and Image 2 (continuous line). These data were obtained with 100 cubes and a $30 \%$ best-frames selection per cube. The filled triangle shows the position of HD $130948 \mathrm{C}$ (see text for details).

faint companion has negligible impact on the position of the primary photo-center. We also verified that the position of the faint companion in the filtered image (Image 2) is possibly altered by the process of spatial filtering by less than 0.2 pixels (or $0.007^{\prime \prime}$ ) in average. The advantage of measuring the BD position in the filtered image is to benefit from an enhanced detection, removing the limitation of a poor signal-to-noise ratio. The two-dimensional Gaussian fit provided an estimate of the relative positions of the two components of $2.561^{\prime \prime} \pm 0.007^{\prime \prime}$ at epoch 2008.56 (46.5 AU at $18.17 \mathrm{pc}$ ), slightly closer compared to the separation of $2.64^{\prime \prime} \pm 0.01^{\prime \prime}$ at epoch 2001.15 found by Potter et al. (2002). The relative orientation of HD 130948 BC with respect to the primary is $\mathrm{PA}=102.7^{\circ} \pm 0.15^{\circ}$, to be compared with $\mathrm{PA}=104.5^{\circ} \pm 0.5^{\circ}$ found by the previous authors. Assuming the distance revised by Dupuy et al. (2009) to $d=18.17 \pm 0.11 \mathrm{pc}$, these measurements may indicate an orbital motion around the primary, with a projected physical separation decreased by $1.43 \pm 0.22 \mathrm{AU}$ in $7.4 \mathrm{yr}$ and a PA change by $1.8^{\circ} \pm 0.55^{\circ}$ in the same period. These values are also agree with the astrometric data provided by Geißler et al. (2008) in their mid-infrared observation of the HD 130948 system at epoch 2006.51. This change corresponds to an approximate orbital velocity projected onto the sky of $\sim 1 \mathrm{~km} \mathrm{~s}^{-1}$, to be compared with an unprojected orbital velocity of $\sim 4.6 \mathrm{~km} \mathrm{~s}^{-1}$ assuming a circular orbit of radius $46.5 \mathrm{AU}$ and a stellar mass of $1.11 M_{\odot}$ for HD 130948 A. The position of the object has been also measured in the image from the May observations. Although the estimate is less precise $\left( \pm 0.015^{\prime \prime}\right.$ and $\left.\pm 0.3^{\circ}\right)$, we find consistent values between the two epochs with $2.56^{\prime \prime}$ and $102.5^{\circ}$ at epoch 2008.41 .

\subsection{Photometry of HD $130948 B C$}

The photometry of the BD companion is extrapolated as a relative measurement to the primary, which is described in Sect. 2.2. From the data obtained with the CAMELOT 


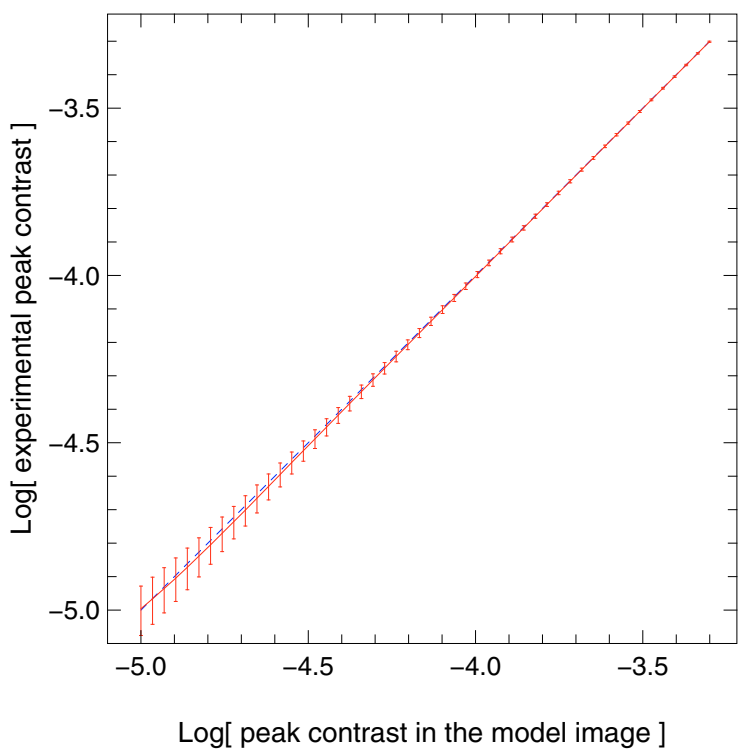

Fig. 3. Evaluation of the impact of the image post-processing on the peak value of a nearby companion. The solid curve with error bars shows, in the $y$-axis, the experimental mean and rms values of the measured peak contrast after image post-processed for various simulated companions contrast at a distance comparable to HD 130948 BC as an input. The $x$-axis shows the input pre-determined model contrast. The dash line is the $y=x$ curve and represents for comparison the contrast values ideally unaffected by the post-processing stage.

instrument, we measured the I-band magnitude of HD 130948 A to be $5.19 \pm 0.03$.

The estimate of the contrast between HD 130948 and the BD companion was obtained by relative comparison with a set of 25 fake companions simulated with comparable separation and brightness in an image model. We compared the peak values of the companions and the fluxes summed in a small aperture of 3 -pixels in radius.

In order to evaluate the effect of the image post-processing on the BD peak value, the contrast of the fake companions in the image model is spanned over 50 values ranging from $5 \times 10^{-4}$ to $10^{-5}$. Each of these 50 models is convolved with our observed PSF of HD 130948 (Image 1) and then filtered, which permits us to directly compare the peak values between the synthesized companion and the original detection in the processed image. The significance of this approach is justified by the small width of the field-of-view $\left( \pm 3^{\prime \prime}\right)$, which is within the typical isoplanatic patch. As a consequence, the speckle pattern in the raw short-exposure is not expected to show significant spatial variations within $\pm 3^{\prime \prime}$, with comparable implications on the PSF of Image 1 . Hence, for each contrast value in the image model, we benefit from 25 independent experimental contrasts, from which mean and rms values are extracted. The statistical dispersion express possible PSF distortion effects in the field-of-view affecting the peak value of the companions (see Fig. 3). From this empirical relationship, the contrast of HD 130948 BC is estimated by interpolation to the measured peak value. As a second approach we also applied the reverse reasoning, which consists in tuning the fake companion contrasts to an arbitrary precision in the image model to match the experimental peak value of HD 13948 BC in the post-processed image. We then derived a new estimate of the mean contrast and corresponding dispersion. Eventually, we applied the same procedures using as input measurements the fluxes summed in the aperture described above.
We are provided in the end with four different estimates of HD 130948 BC contrast with a conservative value of $3.0 \pm 0.3 \times$ $10^{-5}(\Delta I=11.30 \pm 0.11)$ with respect to the $I=5.19$ primary. Note that the peak value method provides a better accuracy $\left( \pm 0.21 \times 10^{-5}\right)$ than the aperture photometry $\left( \pm 0.32 \times 10^{-5}\right)$, in part because in the last case we do not weigh the contribution of each aperture pixel in the way this can be done with optimal extraction techniques. The conversion into magnitudes results into an unresolved BD magnitude $I=16.49 \pm 0.11$ (or $I=17.24$ per component assuming equal brightness for the two components). The combined MKO photometry of the BD from Dupuy et al. (2009) results in the $J=13.2 \pm 0.08$ magnitude, leading to a color index $I-J=3.29 \pm 0.13$ for the unresolved binary. We also verified that the possible elongation by $\sim 30$ mas of the unresolved binary has a negligible effect ( $\lesssim 5$ milli-mag) on the measured photometry.

\section{Discussion}

\subsection{Prospects for the technique}

We applied the wavelet-filtering step to images that do not benefit from AO-assisted or coronographic techniques. We anticipate that the assistance of at least a moderate AO correction able to reduce the halo contribution would improve the achievable contrast at shorter radii in comparison to what we have obtained so far. Hence, we plan to further explore the potential of our approach with observations using the 4.2-m WHT and its AOsystem NAOMI. A fundamental aspect we investigated here to illustrate the potential of the technique is the expected sensitivity limit as a function of the distance to the central star. We selected reasonably clean regions (i.e. unaffected by optical artefacts) of $20 \times 20$ pixels distributed over more than 10 azimuthal positions, centered at $3^{\prime \prime}, 2^{\prime \prime}, 1^{\prime \prime}$, respectively, from the center of Image 2. We then estimated an average of the spatial standard deviation as a function of the number of co-added cubes. The result is illustrated in Fig. 4 with the evolution of normalized $S N R$ as a function of the number of co-added cubes. The bottom plot shows a regular increase of the normalized SNR following a $N^{-0.4}$ power law, where $N$ is the number of co-added cubes. Closer to the star, the plots at $2^{\prime \prime}$ and $1^{\prime \prime}$ obtained with the current data indicate that a relatively high $S N R$ is quickly obtained already after $\sim 10$ cubes, while only slowly increasing afterwards compared to the $3^{\prime \prime}$. Although it is difficult to derive a clear and decisive trend with the current data alone, these plots together with the filtered images presented in this work suggest that as close as $1^{\prime \prime}-2^{\prime \prime}$ the sensitivity is limited by residual speckles, which dominate the image as for traditional AO. Hence, increasing the number of cubes under this regime does not provide a significant gain. This effect should become limited to smaller separation with telescopes larger than the 2.5-m NOT. The small drop in the top plot of Fig. 4 around $N=35$ is possibly caused by an unexpected increase of the speckle noise contribution in the corresponding images. Because it is increasingly dominated by speckle noise at short distance from the star, a further study of the speckle pattern statistics would be required, which is not an objective of the current paper though.

\subsection{Color}

We placed our color measurement in the context of L-type brown dwarfs available photometry (Liebert \& Gizis 2006). In Fig. 5 we reproduce a plot from Liebert \& Gizis (2006) giving the $I_{\mathrm{c}}-J_{\mathrm{MKO}}$ color versus spectral type for a sample of late $\mathrm{M}$ and $\mathrm{L}$ dwarfs, 

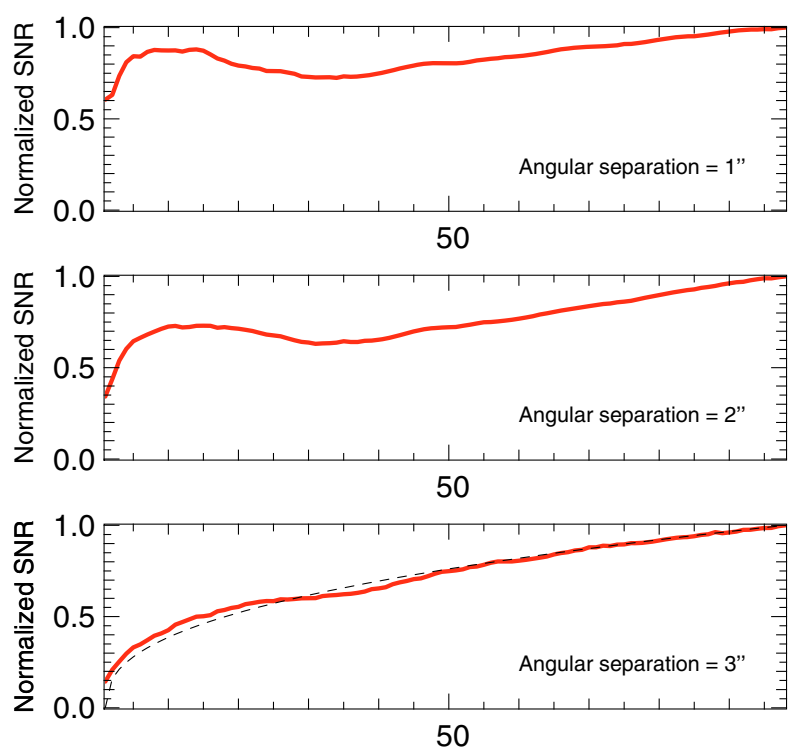

number of cubes

Fig. 4. Normalized $S N R$ as a function of the number of co-added cubes used for the final post-processed image. The curves were obtained by computing the spatial rms in a $20 \times 20$ pixels box at $3^{\prime \prime}, 2^{\prime \prime}$ and $1^{\prime \prime}$ from the primary. Recall that in the present data one cube is composed of 1000 images with $30 \mathrm{~ms}$ exposure from which the $30 \%$ "best" (brightest speckle) images are selected. We overplotted the power-law that best reproduce the experimental data for $3^{\prime \prime}$ separation, namely a $N^{-0.4}$ law (dotted line). The $x$-axis starts at value 0 , and each sub-interval represents 5 cubes.

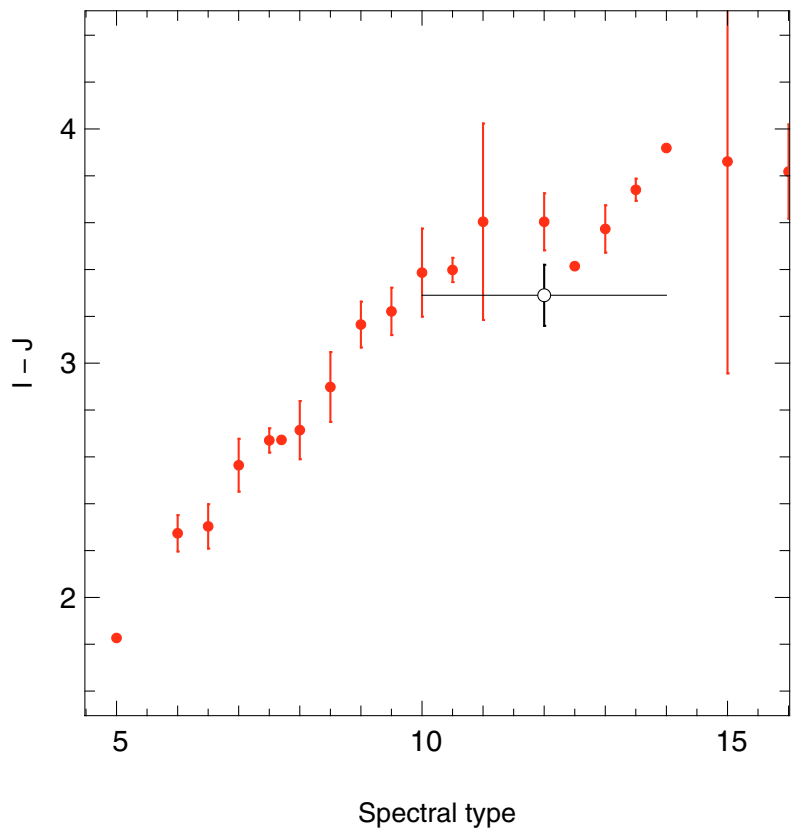

Fig. 5. $I_{\mathrm{c}}-J_{\mathrm{MKO}}$ color versus spectral type reproduced from Liebert \& Gizis (2006) for late M and L dwarfs. Spectral types 5 to 15 corresponds to M5 to L5. Error bars in the photometry represent the measurement dispersion (rms). Our measurement at the 1- $\sigma$ confidence level of HD 130948 BC color is shown as open circle.

after conversion from the 2MASS to the MKO photometric system. Our first measurement of the integrated $I_{\mathrm{c}}$ magnitude of the HD 130948 BD companion allows us to compare it with existing data. We assume for HD 130948 BC a spectral type L2 \pm 2 inferred by near-infrared spectroscopy by Potter et al. (2002). The
$I-J$ color mean and dispersion in the estimated spectral type interval for the Liebert \& Gizis (2006) sample equal $3.58 \pm 0.17$. Within the \pm 2 sub-class uncertainty, the given sample shows only one L dwarfs over 21 objects with bluer color $(I-J=3.19)$ than HD 130948 BC, and classified L0. Hence, this suggests that our object clearly lies in the blue region of the color distribution for this spectral range. This trend becomes even stronger if we place our measurement in the context of the work by Goto et al. (2002), who classified HD 130948 BC as L4 \pm 1 . A similar behavior is also found when placing our color measurement in the $I-J$ versus spectral type plots in Dahn et al. (2002) and Bihain et al. (2010), where typical colors $\sim 3.4-3.6$ are found for L2 dwarfs. The field dwarf 2MASSW J1841086+311727 (L4type, $I_{\mathrm{c}}-J \approx 3.2$, not plotted in Fig. 5) is another known case of spectroscopically reported as an L4 dwarf, although it appears too blue even for an early L (Kirkpatrick et al. 2000). Eventually, our BD object was placed in the $J-K$ versus $I-J$ plot of Liebert \& Gizis (2006) using $J-K \sim 1.6$ measured by Dupuy et al. (2009). In this plot, HD 130948 BC appears consistent with an L2 spectral type, although it lies slightly off the bulk of the L2 BD distribution, suggesting that the object may be peculiar in that diagram as well. Considering this trend, we intend to provide some qualitative explanations to the relative blue color of our object, keeping in mind that further observations would certainly help investigating them.

As suggested by Marley et al. (2002), a possible reason is that for late L dwarfs, the shorter wavelength bands are influenced more by the wings of potassium and other alkali gas opacities.

Also, the metallicity of HD 130948 was measured by Valenti $\&$ Fischer (2005) to $\mathrm{Fe} / \mathrm{H}=0.050 \pm 0.030$, i.e. solar metallicity, thus presumably the same value can apply to the $\mathrm{BD}$ companion. L-type objects in the Pleiades, with metallicity comparable to the Sun, do not present any remarkable bluer $I-J$ colors (Bihain et al. 2010), hence metallicity can probably be ruled out as a cause of the observed effect.

Low surface gravity could also result in a bluer $I-J$ color owing to a weaker potassium line (Kirkpatrick et al. 2008). Because our measured $I-J$ color for HD 130948 is slightly smaller than the average $I-J$ color in the $\sim 120$ Myr Pleiades (cf. Fig. 5 in Bihain et al. 2010), we may have an indication for an even younger age of HD 130948 in comparison to the $0.1-1 \mathrm{Gyr}$ range of age proposed by Dupuy et al. (2009). Also, weatherlike phenomena in the BD atmosphere (Bailer-Jones \& Mundt 1999; Goldman et al. 2008) could cause flux time-variability resulting in a higher dispersion of the $I-J$ color. This case may be explored with repeated monitoring observations of the object, which could be possibly conducted using the observational technique investigated here. Alternatively, stable differences in the surface cloud coverage or in the atmosphere dust properties could alter the measured $I-J$ index.

An additional hypothesis, related to dust in the neighborhood of HD 130948 BC system, could be formulated as the effect of stellar radiation scattered by a residual of dusty disk around the BD companion, with an increased efficiency of the phenomenon toward optical wavelengths (Kalas et al. 2007). Using modeling tools such as the debris disk radiative transfer simulator ${ }^{1}$ (DDS, Wolf \& Hillenbrand 2005), we were able to assess that a small-size "circum-brown dwarf" disk, with a $\sim 10^{-7} M_{\oplus}$ mass and composed of small $(\sim 0.2 \mu \mathrm{m})$ grains confined in an $\sim 2 \mathrm{AU}$ region in radius around the $\mathrm{BC}$ companion, would produce a $I-J$ variation of $\sim 0.2$ mag towards bluer colors. However, the

1 www1.astrophysik.uni-kiel.de/dds/ 
effective extent and geometry of such a disk can clearly not be constrained with these current data. More analytical and observational tests (e.g. focusing on searches for small mid-IR excess for this object) should indicate if such a compact dusty disk would survive in a relatively old multiple system, and how the binary nature of HD $130948 \mathrm{BC}$, with a $\sim 2.2 \mathrm{AU}$ semi-major axis (Dupuy et al. 2009), or even the interaction with the primary would dynamical sculpt the dust distribution into a circum(substellar)binary (Artymowicz \& Lubow 1994) and/or circum(sub)stellar (Mathieu 2007) component. Note that whether this hypothesis is plausible or not the presence of a disk would not be uncommon in substellar objects (Zapatero Osorio et al. 2007; Luhman et al. 2009).

\section{Conclusions}

This work addresses the question of high contrast in optical speckle imaging in the context of substellar objects, in a spectral domain where conventional AO systems present modest performances for long-exposure imaging. We show that the luckyimaging approach has a significant potential to detect faint and close companions to bright stars, which can be improved with an additional stage of post-processing based on the image wavelet transform. We successfully applied this approach for the first time to the system HD 130948 with a clear detection in the $I_{\mathrm{c}}$ filter of the BD binary companion HD 130948 BC at $\sim 2.5^{\prime \prime}$ using the $2.5-\mathrm{m}$ NOT telescope, and without any coronographic system. The BD companion is spatially unresolved because of a too small physical separation (30 mas) at the time of our observations, which is below the telescope resolution at this wavelength. The relative contrast at this distance is estimated to $\Delta I=11.30\left(\sim 3.0 \times 10^{-5}\right)$. At $1^{\prime \prime}$, the point-source detectability is estimated at $\Delta I \sim 10$ in the neighborhood of a bright star like HD 130948 A, making the proposed approach a powerful technique to obtain high-resolution and high-contrast photometry even with small class telescopes. Considering these results, we hopefully plan a positive extension of this approach to larger and AO-assisted medium class telescopes (Law et al. 2009) or eventually to coronographic imaging system. Alternatives to substellar programs could also be considered for science cases where optical imaging is an asset, as for instance for the search for white dwarf companions to bright stars.

Acknowledgements. L.L. is funded by the Spanish MICINN under the Consolider-Ingenio 2010 Program grant CSD2006-00070: first Science with the GTC (www.iac.es/consolider-ingenio-gtc). We wish to thank our anonymous referee who helped us to significantly improve the manuscript. Authors thank support astronomer R. Barrena and the IAC maintenance team. Based on observations made with the Nordic Optical Telescope in the Spanish Observatorio del Roque de los Muchachos of the IAC. This work made use of Yorick (www . maumae.net/yorick), and of the SIMBAD database operated at CDS, Strasbourg, France.

\section{Appendix A: Comparison and details of different image processing algorithms}

We employed three different algorithms to enhance the detection of HD 130948 BC. For all three options, the filters are applied recursively in order to improve the companion detection ( $\sim 3$ iterations, see below). These algorithms are:

$\star$ a median box filter, $I=I$-median $(I, N)$, where $N$ is the size of the median box filter, followed by a convolution with Gaussian kernel to filter out pixel noise;

$\star$ an unsharp mask filter, $I=I-I * g$, where $g$ is a Gaussian kernel, followed by pixel noise filtering;
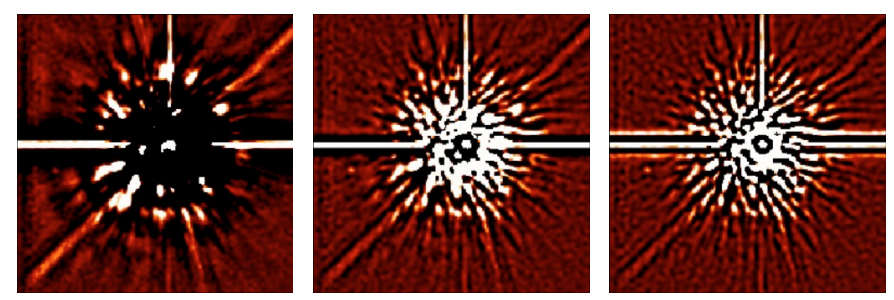

Fig. A.1. Effect of the recursivity in the image filtering process. This series shows our resulting filtered image of the HD 130948 system after one (left), two (center) and three (right) iterations of the wavelet algorithm used in this case.

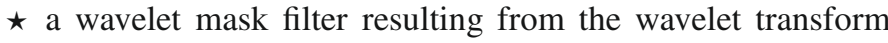
of the image. As a multi-resolution decomposition of the original image, this transform provides a set of filtered images from which can be selected the best trade-off between the spatial resolution and the SNR. The basic algorithm used in this work is largely inspired from the wavelet "à trous" procedure written in C/Yorick language and part of the Yeti package (see www-obs.univ-lyon1.fr/ labo/perso/eric.thiebaut/yeti.html). We apply to the dataset $a$ the function WT( $a$, ORDER), where WT is the wavelet transform described hereafter in the one-dimensional case and extendable to the two-dimensional images for our work. The parameter ORDER gives the maximum degree of decomposition in the multi-resolution analysis. Below, $i$ is the intermediate decomposition degree (also called order) and $1 \leqslant i \leqslant \mathrm{ORDER}+1$. By applying the operation $b=\mathrm{WT}(a$, ORDER), $b$ becomes a cube of images of the same size as $a$ and with its third dimension equal to ORDER +1 , as explained hereafter. The image $b_{\mathrm{i}}$ of intermediate order $i$ is given by

$$
\begin{aligned}
& a_{1}=a \\
& b_{i}=a_{i}-a_{i+1} \\
& a_{i+1}=T\left(a_{i}, \text { SCALE }=2^{i-1}\right) .
\end{aligned}
$$

$T$ is a convolution operation by

$a_{i+1}(p)=\sum_{-W \leqslant j \leqslant W} k(j+W+1) \times a_{i}(p+j \times$ SCALE $)$,

where SCALE $=2^{i-1}$. Integer $p$ is the pixel number in the image, "SCALE" and $W$ are the scaling factor and the halfsize of the wavelet kernel $k$ respectively (i.e. $k$ has a length of $2 \times W+1$ pixels in the one-dimensional case). From cube $b$ we selected one filtered image at the optimal order, which represents the best trade-off between the necessary spatial resolution to detect the companion and a good SNR. If required, the selected filtered image becomes the input image $a$ and the filtering process is run recursively, keeping the same value of the ORDER parameter and eventually selecting the same optimal intermediate order. The reason why this process is run recursively - or iteratively - is to achieve a higher level of suppression of remaining "low spatial frequency structures" in the image, as illustrated in the sequence of Fig. A.1. We verified empirically that there is generally no significant gain after a maximum of three iterations.

The wavelet kernel is support-limited and was empirically optimized for this work to a $5 \times 5$ pixels square (i.e. $W=2$ ) with a two-dimensional distribution given by $[[1,1,1,1,1],[1,3,3$, $3,1],[1,3,8,3,1],[1,3,3,3,1],[1,1,1,1,1]]$.

In order to evaluate the effects of each algorithm, we created 10 fake companions with similar properties to HD 130948 BC 


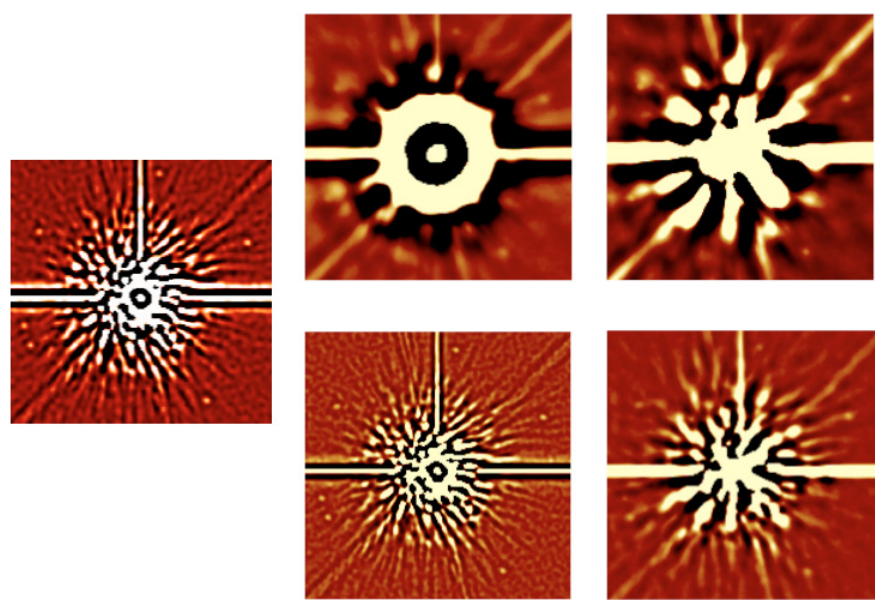

Fig. A.2. Comparison of the different algorithms used here. The intensity scale was optimized to detect the fake companions. Ten individual companions have been simulated for the tests. Only five are displayed here. All presented images have undergone three iterations of the tested filtering algorithms. Left: wavelet algorithm used in this work. Upper-middle: $20 \times 20$ pixels Gaussian kernel filtering followed by $3 \times 3$ Gaussian smoothing to remove pixel noise. Lower-middle: $5 \times 5$ pixels Gaussian kernel filtering followed by $3 \times 3$ Gaussian smoothing to remove pixel noise. Upper-right: $20 \times 20$ pixels median filtering followed by $3 \times 3$ Gaussian smoothing to remove pixel noise. Lower-right: $5 \times 5$ pixels median filtering followed by $3 \times 3$ Gaussian smoothing to remove pixel noise.

and radially distributed around HD 130948 A. As an additional test, this permitted us to discard possible algorithm artefacts that would have led to a false detection of HD 130948 BC.

The imaging results are shown in Fig. A.2. We initially implemented the two first algorithms using a kernel with a $F W H M$ of 4-5 times the original PSF FWHM. In both cases this led to an excessive smoothing of the image preventing us from detecting all of the companions and in particular HD 130948 BC (see Fig. A.2, upper-middle and upper-right panel). Reducing the convolution kernel to a size comparable with the PSF FWHM significantly improved the result, although the median box filter does not reach a satisfactory level. On the contrary, the iterative unsharp mask filtering using a smaller kernel permits us to detect all companions, included HD 130948 BC. This suggests that apart from the multi-resolution decomposition - which is useful to identify a close-to optimal kernel size - unsharp mask filtering is almost identical in terms of point-like source detectability to the wavelet filtering algorithm described above.

\section{References}

Artymowicz, P., \& Lubow, S. H. 1994, ApJ, 421, 651

Bailer-Jones, C. A. L., \& Mundt, R. 1999, A\&A, 348, 800

Bihain, G., Rebolo, R., Zapatero Osorio, M. R., Béjar, V. J. S., \& Caballero, J. A. 2010, A\&A, 519, A93

Boccaletti, A., Moutou, C., Mouillet, D., Lagrange, A., \& Augereau, J. 2001, A\&A, 367, 371

Bouy, H., Duchêne, G., Köhler, R., et al. 2004, A\&A, 423, 341

Burgasser, A. J., Kirkpatrick, J. D., \& Lowrance, P. J. 2005, AJ, 129, 2849

Dahn, C. C., Harris, H. C., Vrba, F. J., et al. 2002, AJ, 124, 1170

Dupuy, T. J., Liu, M. C., \& Ireland, M. J. 2009, ApJ, 692, 729

Geißler, K., Chauvin, G., \& Sterzik, M. F. 2008, A\&A, 480, 193

Goldman, B., Cushing, M. C., Marley, M. S., et al. 2008, A\&A, 487, 277

Goto, M., Kobayashi, N., Terada, H., et al. 2002, ApJ, 567, L59

Kalas, P., Fitzgerald, M. P., \& Graham, J. R. 2007, ApJ, 661, L85

Kirkpatrick, J. D., Reid, I. N., Liebert, J., et al. 2000, AJ, 120, 447

Kirkpatrick, J. D., Cruz, K. L., Barman, T. S., et al. 2008, ApJ, 689, 1295

Landolt, A. U. 1992, AJ, 104, 340

Law, N. M., Hodgkin, S. T., \& Mackay, C. D. 2006a, MNRAS, 368, 1917

Law, N. M., Mackay, C. D., \& Baldwin, J. E. 2006b, A\&A, 446, 739

Law, N. M., Mackay, C. D., Dekany, R. G., et al. 2009, ApJ, 692, 924

Liebert, J., \& Gizis, J. E. 2006, PASP, 118, 659

Liu, M. C., Fischer, D. A., Graham, J. R., et al. 2002, ApJ, 571, 519

Luhman, K. L., Mamajek, E. E., Allen, P. R., \& Cruz, K. L. 2009, ApJ, 703, 399

Marley, M. S., Seager, S., Saumon, D., et al. 2002, ApJ, 568, 335

Mathieu, R. D. 2007, in IAU Symp. 243, ed. J. Bouvier, \& I. Appenzeller, 315

Oscoz, A., Rebolo, R., López, R., et al. 2008, in SPIE Conf., 7014

Potter, D., Martín, E. L., Cushing, M. C., et al. 2002, ApJ, 567, L133

Stassun, K. G., Mathieu, R. D., \& Valenti, J. A. 2006, Nature, 440, 311

Valenti, J. A., \& Fischer, D. A. 2005, ApJS, 159, 141

Wolf, S., \& Hillenbrand, L. A. 2005, Comput. Phys. Commun., 171, 208

Zapatero Osorio, M. R., Lane, B. F., Pavlenko, Y., et al. 2004, ApJ, 615, 958

Zapatero Osorio, M. R., Caballero, J. A., Béjar, V. J. S., et al. 2007, A\&A, 472, L9 